\title{
The effectiveness of the tests used for predicting intubation difficulty and the use of McGrath Videolaryngoscope in cases BMI $>30 \mathrm{~kg} / \mathrm{m}^{2}$
}

\author{
Basar H., Baltaci B., Cakirca M., Bedevi R.. \\ Ankara Training and Research Hospital, Dept of Anaesthesiology, Ankara, Turkey
}

\section{Background and Goal of Study}

The incidence of difficult airway is high in obese patients due to large neck circumference, small mouth opening, short sternomental and thyromental distances as well as the effect of obesity related systematic diseases on morbidity. It is stated that videolaryngoscopy provides a better glottic view increases the success rate of endotracheal intubation.

In this study it is aimed to evaluate the effectiveness of the tests which are used to determine the difficulty of intubation and to evaluate the effectiveness of McGrath Videolaryngoscopy in obese patients (BMI $>30 \mathrm{~kg} / \mathrm{m}^{2}$ ) undergoing elective surgery.

\section{Material and Methods}

ASA I-III one hundred patients, aged above 18 years, which are undergoing elective surgery and endotracheal intubation was included into the study with informed consent after approval from Ankara Training and Research Hospital Training Planning Committee.

Mallampati test was evaluated according to Samsoon-Young classification in all cases in the operating room. Required measurements were done after that. Which include height, weight, neck circumference, thyromental distance, sternomental distance and mouth opening. It was also evaluated the association height to neck circumference ratio, height to thyromental distance ratio and neck circumference to thyromental distance ratio with intubation difficulty while the above mentioned measurements were not sufficient to determine difficult intubation.

Cormack-Lehane classification was evaluated with Macintosh Laryngoscope following anesthesia induction and mask ventilation of three minutes. Thereafter mask ventilation was continued. CormackLehane classification evaluated again with McGrath Videolaryngoscope by a second anesthetist and then endotracheal intubation was performed.

Duration of intubation and the number of attempts were recorded. Duration of intubation was accepted as the duration between the time of insertion of McGrath Videolaryngoscope into the mouth and the time of pass of endotracheal tube between vocal cords. Intubation Difficulty Scale (IDS) was used to evaluate the difficulty of intubation. Unable to intubate in three attempts or a duration of 120 seconds was accepted as failed intubation.

Descriptive statistics was showed as mean \pm standard deviations for categorical variables. T-test, Mann-Whitney U test or Kruskal Wallis variance analyses was used for between group comparisons for continuous variables in independent groups. Wilcoxon test was used for continuous variables in inter group comparisons. $\mathrm{P}<0,05$ was accepted as statistically significant. Analysis of data was done in SPSS 15 program.

\section{Results}

Difficult intubation according to IDS scoring (IDS > 5) was not seen in our study. A significant positive correlation was found between Mallampati classification, Cormack-Lehane classification and neck circumference and the duration of intubation. A significant negative correlation was found between height to neck circumference ratio and the mouth opening and the duration of intubation.

\begin{tabular}{|l|l|}
\hline Cormack-Lehane(M) I/II/III/IV & $28 / 50 / 22 / 0$ \\
Cormack-Lehane(VL) I/II/III/IV & $94 / 6 / 0 / 0$ \\
\hline IDS 0/1/2/3 & $28 / 49 / 17 / 6$ \\
\hline Intubation time (sec) & $24,2 \pm 15$ \\
\hline
\end{tabular}

Figure 1. Cormack-Lehane $(\mathrm{M})$ : Cormack-Lehane score with Machintosh blade Cormack-Lehane(VL) : Cormack-Lehane score with Videolaryngoscopy IDS : Intubation Difficulty Score

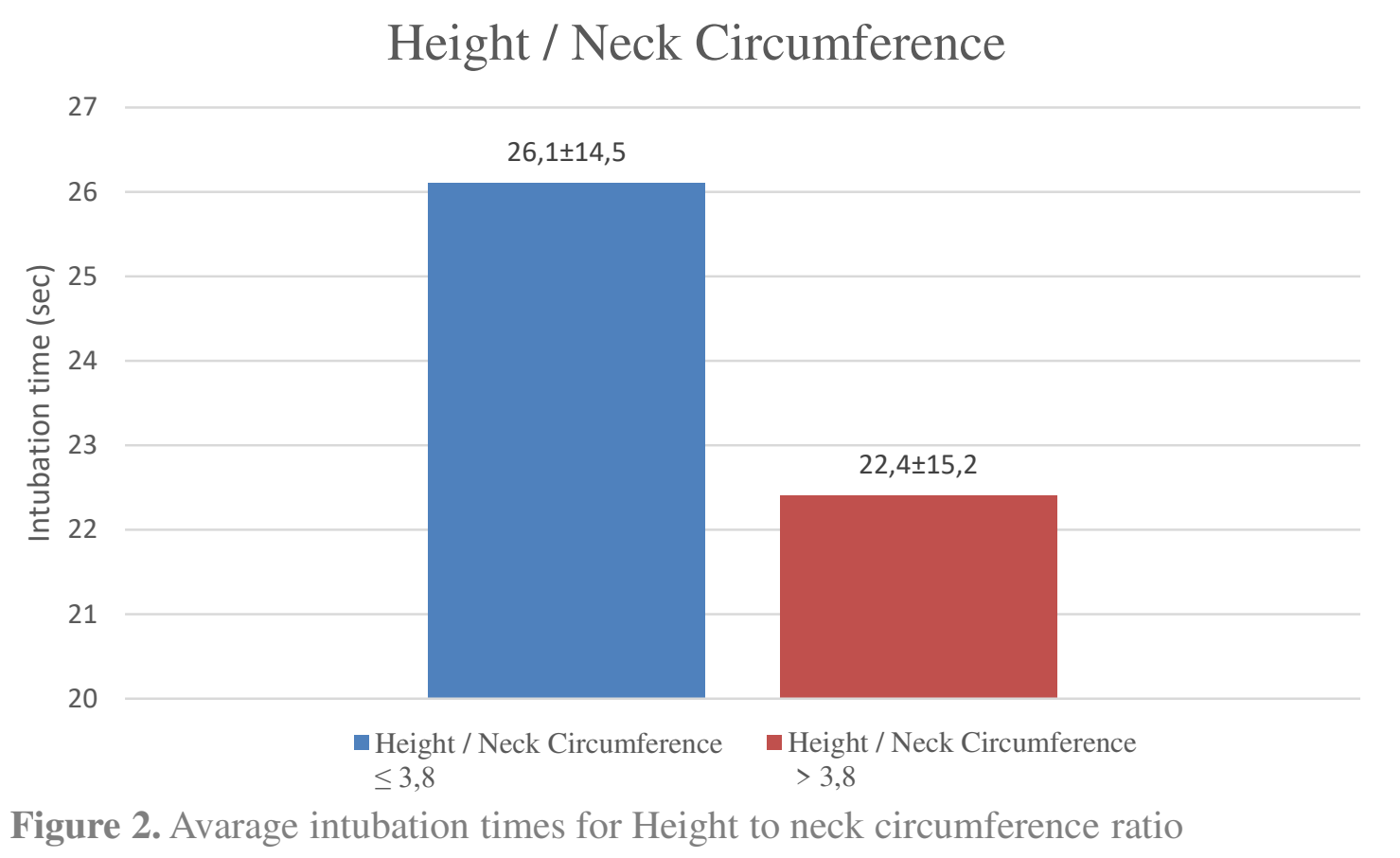

\section{Conclusion}

In this study, it is found that videolaryngoscopy is effective in obese patients. Neck circumference, mouth opening, Mallampati and Cormack-Lehane classification was found as significant parameters for difficult intubation as well as height to neck circumference ratio is a significant criteria for it. It is concluded that it is beneficial to use these parameters for detecting difficult intubation cases in anesthesia practice and more studies needed to test the effectiveness of height to neck circumference ratio for predicting difficult intubation. 